\title{
Redes Sociales y Socialización Afectiva de las Personas Jóvenes: Necesidades Docentes en Educación Secundaria Obligatoria
}

\author{
Social Media and Affective Socialization of Young People: \\ Teaching Needs in Compulsory Secondary Education
}

\author{
Soraya Calvo González * \\ José Luis San Fabián \\ Universidad de Oviedo, España
}

\begin{abstract}
Se analizan las necesidades y la situación de partida que detectamos tras estudiar las opiniones y percepciones que distintos profesionales de la educación tienen acerca del uso que las personas jóvenes hacen de las TIC para relacionarse emocionalmente. Nuestros datos fueron recogidos a partir de la aplicación de entrevistas semiestructuradas a informantes clave y grupos focales. Contamos con una muestra de 30 docentes en activo vinculados a 12 centros educativos de Asturias. El análisis cualitativo de tipo descriptivo y comparativo evidencia que existen importantes disonancias entre la visión del profesorado, la implicación de las familias y la vivencia cotidiana real del alumnado. Las disonancias detectadas son atribuidas a una falta de formación técnica y competencial. Esto se suma al desconocimiento que el profesorado siente y muestra en torno a los modelos de comunicación afectiva en redes sociales de las personas jóvenes. También se muestra una desconexión con los referentes mediáticos culturales y la influencia que estos tienen en la construcción identitaria y en la vinculación a valores, mitos e ideologías del colectivo adolescente. La distancia señalada dificulta el trabajo educativo significativo y la adecuación de contenidos y metodologías a las estrategias reales de interacción en las vivencias cotidianas del alumnado. En línea con los diferentes aspectos de interés y necesidades señaladas, aportamos conclusiones para la mejora de la situación en clave de equidad.
\end{abstract}

Descriptores: Formación de docentes; Tecnología educacional; Joven; Comunicación; Competencias para la vida.

It is analyzed the needs and the starting situation that we detect after studying the opinions and perceptions that different education professionals have about the use that young people make of ITC to relate emotionally. Our data were collected from the application of semi-structured interviews to key informants and focus groups. We have a sample of 30 teachers linked to 12 Asturian secondary schools. The descriptive and comparative qualitative analysis evidences the existence of important dissonances among teachers view, the implication of families and the real daily life of the students. The detected dissonances are attributed to a lack of competence and technical formation. All this adds to the lack of knowledge that teachers feel and show about affective communication models in in social networks of young people. It also shows a disconnection with the media cultural references and the influence of those in identity building and the relation to values, myths and ideologies. This is a handicap to develop significant educational work and to adapt contents and methodologies to real interaction strategies of students. According to different aspects of interest and indicated needs, we provide conclusions to improve the situation in terms of equity.

Keywords: Teacher training; Educational technology; Youth; Communication; Life skills.

*Contacto: calvosoraya@uniovi.es

ISSN: $1696-4713$

www.rinace.net/reice/

revistas.uam.es/reice
Recibido: 1 de noviembre 2017

$1^{\text {a }}$ Evaluación: 17 de diciembre 2017

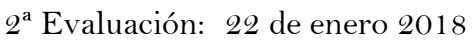

Aceptado: 27 de enero 2018 
Esta investigación ha sido posible gracias a la financiación derivada del programa Severo Ochoa de ayudas predoctorales para la formación en investigación y docencia (BP-12128) gestionadas por FICrT (para el fomento en Asturias de la investigación científica aplicada y la tecnología).

\section{Revisión de la literatura}

Los agentes educativos digitales, en el marco de una sociedad definida por la incorporación de la tecnología en las realidades cotidianas, forman parte de las vidas de las personas y tienen influencia en la manera en la que cada cual concibe el mundo y sus posibilidades de acción (Buckingham y Martínez-Rodríguez, 2013).

Las tecnologías, como medios que reproducen y ejercen poder educativo, son elementos clave de los procesos formativos escolares. Ello pone de manifiesto los límites del conocimiento tradicional en tanto que estos agentes informales superan los ámbitos físicos y de formato de la escuela o universidades, implicando la necesidad de analizar resultados de las interacciones entre esos agentes y las vivencias emocionales de las personas en un contexto primado por lo digital (Serrano-Puche, 2016). Las características de multiplicidad en las representaciones digitales de identidades y/o personalidades, y la manera en que los colectivos ejercen impresión social en los espacios en línea (Wood y Smith, 2005), crean opciones reales en torno a la figura de las redes sociales como contextos mediáticos emergentes con posibilidades educativas (Livingstone et al., 2011).

Tras revisar varios materiales académicos y/o didácticos sobre los usos de las TIC en la infancia y la adolescencia (Almansa, Fonseca y Castillo, 2013; García-Jiménez, López de Ayala-López y Catalina-García, 2013; Domínguez-Fernández, Jaén-Martínez y CeballosGarcía, 2017), detectamos una tendencia general en dos direcciones mayoritarias: una que pretende mostrar conclusiones explicativas y descriptivas, en ocasiones técnicas, sobre usos generalistas sin profundizar en opciones de acción; y otra que contribuye a fomentar el "diseño de estrategias y líneas de actuación a nivel de prevención y control, que favorezcan un uso más saludable de Internet entre los más jóvenes” (Rial et al., 2014, p. 653). ¿Qué otras formas existen de enfrentarnos, desde un enfoque educativo, a la sociedad red tal y como la entiende Castells (2006)?

Este estudio parte del deseo de que la investigación en torno a los usos jóvenes de las TIC tenga traducciones transformadoras de educación mediática para lograr un empoderamiento activo y autónomo en el marco de las relaciones y experiencias tanto personales como académicas. Para ello es imprescindible conocer no solo las realidades vivenciales de las personas jóvenes, sino también las percepciones que el profesorado tiene de las mismas para avanzar hacia un cambio político y metodológico real.

\subsection{Usos jóvenes de las TIC e implicaciones emocionales}

En un entorno sociotecnológico en el que más de 23 millones de personas de 16 a 74 años se conectan a Internet todos los días (ONTSI, 2017), ¿cómo influye internet en la vida social y personal de la juventud española? Esta pregunta, con diferentes matices y perspectivas, es la que se han hecho numerosos artículos a lo largo de estos últimos años de democratización tecnológica. En España, según el INE, hay 34.389 .822 personas mayores de 16 años, de las cuales un 82,7\%, más de 28 millones, han utilizado Internet en alguna ocasión (ONTSI, 2017). De esos 28 millones en torno a 4 son jóvenes entre 16 y 24 años, los cuales se conectan, casi en su totalidad todos los días a Internet. Como dato especialmente llamativo podemos destacar que, según el mismo informe, el número de 
personas de 10 años y más que han accedido a Internet en alguna ocasión supera los 31 millones y medio en el año 2016.

Desde hace ya varios años se viene admitiendo que, para los jóvenes actuales, usar Internet es algo tan natural como para sus referentes adultos lo era ver televisión o escuchar la radio (Bernete, 2009). Además, al ser un colectivo especialmente familiarizado con las tecnologías de la información y la comunicación (Aymerich-Franch y Fedele, 2015), tener en cuenta sus usos, opiniones y críticas en clave de revisión es una buena forma de medir la realidad digital del momento. En línea con estos supuestos, los interrogantes sobre sus usos y consumos (Barrios, 2009; Colás-Bravo, González-Ramírez y de Pablos-Pons, 2013) han sido una dimensión fluctuante y líquida. Las fluctuaciones de las redes sociales mayoritarias y las condiciones cambiantes de acceso determinadas por el mercado de las tecnologías han favorecido un contexto extremadamente variable, el cual debe ser atendido casi constantemente para no perder la visión real de lo que está sucediendo en las pantallas. Así, si hace no más de seis años hablábamos de Tuenti (Sixto, 2011) y MySpace como referentes en lo que a la presencia adolescente se refiere, en la actualidad hablamos de la revolución digital que ha supuesto la irrupción de Instagram (Marcelino, 2015), con todas las repercusiones que estos cambios entre plataformas han tenido en la estructuración de identidades, narrativas e intermediaciones. Consideramos relevante detenernos en el concepto de red social para aclararlo y delimitarlo. Para Campos-Freire (2008), las redes sociales se sustentan en un concepto de comunidad conformada por redes de usuarios que tienen interacción, diálogo y generan dinámicas comunicativas y de conocimiento. Existe un cambio de formato, ya que mientras en la web 1.0 (no social) las personas usuarias asumían principalmente un rol de receptoras, en la web 2.0 existe la posibilidad de producir contenidos, participar del valor del intercambio y colaborar en el desarrollo de la tecnología. Por ello, las redes sociales y la puesta en marcha de su proceso comunicativo generan, en definitiva, un flujo activo de participación. En un entorno digital casi infinito como es Internet llega a ser necesario la distinción entre los diferentes servicios ofertados para así entender cuáles son las posibilidades de usuarios y usuarias en su interacción con las mismas. El objetivo principal de estos espacios bidireccionales es fomentar y fortalecer las relaciones interpersonales, ya sea con aquellas personas que conoces previamente y/o con las que mantienes una relación de amistad, o bien con otras que no forman parte de nuestro círculo de amistades pero que nos interesan por un motivo concreto (atracción, interés, afinidad...). Según Monsoriu (2009), las redes sociales nos permiten, entre otras posibilidades, compartir, etiquetar y comentar vídeos fotos y enlaces, generar discusiones, jugar en línea, enviar mensajes privados, acceder a perfiles de nuestros contactos o tratar de generar nuevas conexiones con personas que no conocemos.

\subsection{Repaso histórico a la presencia de las redes sociales mayoritarias en España}

Uno de los estudios pioneros a gran escala que se desarrolló en España para conocer la situación virtual del colectivo de jóvenes fue el llamado "Informe Generación 2.0" (Sánchez-Burón y Álvaro-Martín, 2010). En este informe, con una muestra de 6.798 adolescentes de entre 12 y 17 años que cursaban Educación Secundaria Obligatoria y Bachillerato en 44 centros educativos públicos, privados y concertados de España, se demostró que el uso de redes sociales ya estaba muy extendido entre la población joven. Únicamente el $4 \%$ de la muestra decía no conocer lo que eran las redes sociales y el $18 \%$ afirmaba conocerlas, pero no utilizarlas. Casi la totalidad de los jóvenes que usaban redes sociales decían conocerlas gracias al boca a boca, siendo la publicidad (o, al menos, la explícita) un elemento aparentemente inútil para la transmisión del consumo de estos 
artefactos tecnológicos. Por otro lado, la gran mayoría de la muestra utilizaba en aquel momento las redes desde hacía menos de tres años. En cuanto a las posibilidades de Internet, los y las adolescentes parecían desconocer otros usos que fueran más allá de la participación en plataformas sociales, ya que la generación de blogs y páginas web son actividades muy poco secundadas por este colectivo. En aquel año 2010, Tuenti era la red social más utilizada, con un aplastante $80 \%$ frente al $14 \%$ de Facebook o el $1 \%$ de Twitter. Los datos sobre la actividad siguen una coherencia respecto a los datos anteriores, siendo Tuenti la red con más actividad. A pesar de que Facebook era entendida como una red social minoritaria en su uso, más de la mitad de la muestra había abierto un perfil en esta red social, aunque no le diera actividad posteriormente. Respecto a la frecuencia de los usos, más de la mitad de la muestra que participo en el estudio accedían a sus perfiles en las redes diariamente, y la intención de uso principal de la mayoría era orientada a mantener el contacto con amigos/as y personas conocidas. Las fotos, los mensajes privados y los comentarios a las fotos de contactos son las actividades más populares dentro de ese uso principal que se desprende de la investigación. Otros de los datos más relevantes de la investigación son aquellos que hacen alusión a la tendencia general de añadir y mantener como contactos solo a personas a las que se conoce previamente en persona, desterrando la idea de que las redes sociales son espacios para hablar con desconocidos/as. En aquel momento no se veían evidencias que relacionaran las calificaciones negativas en forma de suspensos con un mayor acceso a las redes, ni tampoco parecía que las redes sociales supusieran una actividad que reemplazara a otras tales como salir con los amigos, dormir o hacer deporte. El tiempo offline se valoraba positivamente por encima del tiempo online, y no parecían existir significativas brechas de comunicación con amistades y familias debido al uso de las tecnologías, por ejemplo, no enterarse de lo que pasó en un grupo por no usar determinada aplicación. Finalmente, existía una percepción destacable de los riesgos derivados del uso de las redes sociales, asegurando más de un $70 \%$ de los y las participantes la toma de medidas activas para proteger su privacidad.

Desde el 2010 han cambiado muchas cosas: en primer lugar, la manera en la que se aborda el tema de estudio. Si bien el estudio "Informe Generación 2.0" supuso un acercamiento muy interesante a la realidad digital de adolescentes, no abordó el uso del smartphone como componente distintivo del acercamiento a las redes, y tampoco se centró en las características de las interacciones, lo cual podría haber dado una visión más realista del panorama 2.0 del momento. En el contexto de la publicación de este informe, un estudio de José Sixto (2010) defendía que Facebook comenzaba a ganarle la batalla a Tuenti en número de usuarios, sin especificar las edades de los mismos, pero abriendo una nueva perspectiva de análisis que sería sumada y apoyada por otros autores (Almansa, Fonseca y Castillo, 2013; Caldevilla, 2010). Hasta ese momento Tuenti supuso una revolución en el panorama mediático español.

A pesar de que Facebook era la red social con más presencia en el mundo occidental, en el caso de nuestro país los usos iniciales entre las personas jóvenes no siguieron esa tendencia general, y fue la red social española la que verdaderamente introdujo el concepto en la vida real y diaria de los y las jóvenes. Podría decirse que Tuenti fue la primera red social mayoritaria y popularizada en nuestro país, ya que hasta el momento la herramienta web de comunicación más utilizada por los y las jóvenes era el Messenger de Microsoft (Graner et al., 2007). 
Tuenti fue una red social dirigida a un público adolescente y universitario, mayor de 14 años, y que gozó de una especial viralidad. Para poder abrirse un perfil en Tuenti era necesaria una invitación previa por parte de una de las personas usuarias de la red y posteriormente un registro. Cada usuario/a disponía de un número limitado de invitaciones. Para esta red, "esa garantía de protección de usuarios falsos o extraños es uno de los elementos de valor que la red potencia” (Sixto, 2011, p. 4). Tuenti ofrecía posibilidades de comunicación muy parecidas a las de otras redes como Facebook: eventos, subida de fotografías y gestión de álbumes, "peticiones de amistad", comentarios, estado personal, un chat para hablar en privado, posibilidad de gestionar la privacidad (aunque de una forma muy limitada), publicación de datos personales tales como el cumpleaños etc. Como elementos diferenciadores, Tuenti también ofrecía otras opciones muy orientadas a ese público objetivo adolescente (Sixto, 2011): un contador de visitas que mostraba el número pero no los datos personales de aquellos y aquellas que accedían a cada perfil, interacción con el usuario en el momento de acceso mediante preguntas sobre las actividades que había realizado en los últimos días, un blog personal o un espacio para añadir en el perfil las "zonas de fiesta", el colegio o instituto donde estudió o estaba estudiando el/a usuario/a, etc.

La hegemonía de Tuenti como red social mayoritaria fue difuminándose con el tiempo, siendo Facebook su principal competencia. Así, si en el informe "Generación 2.0" veíamos a Tuenti como la opción claramente ganadora, en los estudios posteriores (GarcíaJiménez, López de Ayala-López y Catalina-García, 2013; Reguillo, 2012; Rial et al., 2014) las cifras de participación de jóvenes en las dos redes sociales fueron asimilándose cada vez más, desapareciendo esa clara superioridad. A pesar de que Facebook parecía perfilarse cada vez más como una opción relevante para este colectivo, algunas voces nos alertan de la falta de significatividad que los y las menores de España sintieron respecto a la misma: Facebook es considerada por ellos una red social para adultos, de forma que, aunque muchos y muchas tengan perfil en esta red social, apenas la utilizan para comunicarse y relacionarse con sus pares (Merino, 2011). Si Facebook está presente pero no es utilizada diariamente, ¿¿cuál es la red social más utilizada por los y las jóvenes en la actualidad?

Para conocer las tendencias generales de uso de redes sociales, y tomando de referencia el total de la población en España, podemos atender a los datos de la última edición de la Encuesta AIMC (2017) a Usuarios de Internet, Navegantes en la Red:

- Más del 98\% de las personas se conectan todos los días a Internet. Casi el 80\%, además, parece evidenciar una tendencia a conectarse diariamente a las redes sociales.

- Facebook es, actualmente, la red social más utilizada, por delante de Twitter. Instagram se muestra en imparable subida.

- Más del 53\% de los y las participantes declaran estar muy preocupados por la privacidad de su intimidad en las redes sociales.

- El uso prioritario del smartphone es el vinculado a la comunicación. Más del 91\% de las personas internautas así lo defienden.

Los datos respecto al uso de las redes sociales parecen presentar cierta controversia. Tras la desaparición en 2016 de la red social Tuenti, aún existen estudios que sitúan a Facebook como referente de la actividad en redes de la juventud en España (García-Martín y GarcíaSánchez, 2015), pero los reportes de organizaciones vinculadas a la comunicación parecen 
destacar un panorama muy diferente. El VI Estudio Redes Sociales (IAB, 2017) muestra un desuso muy evidente de aplicaciones como Google+ o Snapchat y desaparecen redes anteriormente estudiadas como Badoo. En sus datos orientados a visibilizar la realidad adolescente se muestra una subida clara de Instagram y YouTube y una bajada en el caso de Facebook, en los tres casos de manera muy significativa respecto al mismo estudio en años anteriores.

Con Instagram se llega a hablar de migración tecnológica (Marcelino, 2015). Instagram es una red social muy simple, con pocas utilidades y que presenta como objetivo la publicación de imágenes, su retoque y su etiquetado. No tiene muchas posibilidades para gestionar la privacidad, ya que tampoco se pueden gestionar contactos ni publicaciones de manera individualizada y personalizada. La fuerza de esta red recae no solo en su simplicidad, sino también en la potencialidad de la imagen como contenido relevante y emocional para el colectivo de personas adolescentes (Arriaga y Marcellán Baraze, 2016; Ballesteros y Megías, 2015). Instagram, al contrario de lo que se desprende del uso de otras redes, como Twitter, que son consideradas "complementos" de Facebook (Madden, Lenhart y Cortesi, 2013), se utiliza de manera individual y con importancia propia (Marcelino, 2015). Si bien aún no existe gran producción académica al respecto del uso de Instagram en España como objeto de estudio debido probablemente a la rapidez con la que están variando las tendencias, podemos aludir a diferentes reportes extranjeros que comienzan a interesarse por esta red como espacio de socialización en línea ( $\mathrm{Hu}$, Manikonda y Kambhampati, 2014; Salomon, 2013). Nos gustaría destacar el estudio llevado a cabo por Eunji Lee y su equipo (2015), que señala cinco motivos sociales y psicológicos principales que parecen conducir hacia el uso de esta red social: la interacción social, la recopilación de los momentos vitales importantes, la expresión personal, las tendencias escapistas y cotillear desde el anonimato.

\subsection{De las redes sociales a la mensajería instantánea: WhatsApp y el contexto del teléfono móvil}

La convivencia entre las variaciones de uso en torno a las redes sociales y la estabilidad de WhatsApp como aplicación de mensajería instantánea dominante en todos los estudios aludidos (AIMC, 2017; IAB, 2017) nos sitúa en un nuevo marco de análisis. Las redes sociales parecen ser el día a día, pero WhatsApp es el minuto a minuto. El uso cada vez más cotidiano de teléfonos móviles con acceso a Internet por parte de las personas jóvenes ha definido un nuevo tipo de acceso a las redes más continuo, más cercano y al alcance de la mano (Barujel, Varela y Rodes, 2017; Garmendia et al., 2016). Es por ello que si bien las redes sociales se convierten en herramientas para conocer, publicar y compartir; las aplicaciones de mensajería instantánea como WhatsApp son consideradas como la principal vía de comunicación directa con el entorno cercano, las amistades y la familia.

WhatsApp es la aplicación de mensajería más descargada en nuestro país, así lo confirman los estudios "AppDate" que desde el año 2011 pretenden dibujar el estado y uso de las Apps. La Encuesta AIMC a Usuarios de Internet, Navegantes del 2017 señala que el 93\% de las personas participantes utilizan WhatsApp como servicio de comunicación directa, siendo esta cifra claramente superior que la de quienes usan el chat de Facebook (52\%) u otras aplicaciones como Skype (35\%) o Telegram (22,1\%).

Es útil comparar WhatsApp con sus antecesores, los SMS, para obtener una retrospectiva de lo que el cambio de la tecnología ha supuesto para la transformación de la comunicación resultante. WhatsApp no ha sido simplemente un sustituto: ha reformulado el concepto 
de mensaje móvil, superando el componente puramente textual (Gómez del Castillo, 2017). Ahora la comunicación textual a través de estos dispositivos es más barata, más cotidiana, percibida como más inmediata, y es expansiva. Además, es más fácil gestionar los datos que se comparten con los demás ya que la propia aplicación simplifica en gran medida la identidad digital frente a otras redes sociales como Facebook: foto de perfil, nombre y estado, sin posibilidad de que otras personas te etiqueten, te comenten o te nombren (Caro, 2015). La interacción a través de WhatsApp permite una gestión más eficaz de los grupos, siendo más útil para planear, coordinar y crear debate o discusión (Church y de Oliveira, 2013). Esta aplicación permite diálogos más fluidos y sociales, y además pone en juego elementos que enriquecen el lenguaje en formato texto como imágenes y textos adjuntos, incorporando también emoticonos, lo que permite incluir en las conversaciones elementos simbólicos (Church y de Oliveira, 2013; Cuadrado et al., 2014; Qiu et al., 2015). Como últimos rasgos destacados, refuerza la idea de "pertenencia a grupo" gracias a su efecto multiplicador de los mensajes y las conexiones. "WhatsApp, conceptualmente y sobre todo desde la práctica, se entiende como el recurso para radiartelegrafiar la vida, relatar, narrar, expresarse, citarse, etc.” (Reig y Vílchez, 2013, p. 133).

En esa transformación también existen hándicaps que pueden desvirtuar ese proceso comunicativo y marcar barreras en su efectividad. La comunicación virtual es reconocida "como facilitadora del acercamiento, pero al ser más impersonal es igualmente más demandante de esfuerzo para complementar el sentido de los mensajes" (Rubio-Romero y Perlado, 2015, p. 90). Sumado a ello se hace hincapié sobre la sensación de obligación de tener que atender a las necesidades de comunicación de las demás personas en formato 24/7, 24 horas al día y 7 días a la semana, y la amenaza a la propia intimidad, lo que deriva en una pérdida de control de la situación social de la persona receptora al ofrecer la propia aplicación información sobre tiempos y conexiones (Caro, 2015).

La predisposición a usar el teléfono móvil como principal punto de conexión a redes sociales y demás aplicaciones 2.0 lo convierte como un objeto relevante para entender más sobre los usos tecnológicos de la gente joven. El triunfo de esta tecnología parece derivarse de varias características: instantaneidad, portabilidad, manejabilidad, sus posibilidades multifunción y multiformato, su carácter intuitivo y el coste del aparato y de la conectividad, que normalmente es asumido por las personas adultas (padres, madres, etc.). El teléfono móvil, en su dimensión instrumental, asume múltiples roles: comunicación, expresión, ocio e información. "Lo atractivo del smart es su multifunción, que posibilita muchas cosas, multiefectividad y multiafectividad” (Reig y Vílchez, 2013, p. 132).

Las motivaciones sociales que refuerzan el uso del teléfono móvil están fuertemente vinculadas a las relaciones sociales que se establecen a partir de él. Cuesta y Gaspar (2013) aluden a ellas de la siguiente manera: en primer lugar, hablan de la motivación de búsqueda de información, que consiste en la fuerte necesidad de buscar y localizar información en su entorno contextual; y en segundo lugar, detectan el deseo de búsqueda de contactos para establecer vínculos y relaciones. Ese apego a la herramienta, en ocasiones tratada desde la idea de la posibilidad adictiva y su engagement generalizado (Chóliz, 2012), perfila un plano social en el que es extraño encontrarse con un o una adolescente que no disponga de teléfono móvil debido, entre otras cosas, a que sus procesos de socialización no se entienden alejados del uso de estos recursos. Las posibilidades y los hándicaps que hemos comentado acerca de WhatsApp en párrafos anteriores podrían hacerse extensibles a los 
que nos encontramos al utilizar teléfonos móviles de última generación, con una clara salvedad: al disponer de un mundo de apps casi infinito, las posibilidades y los hándicaps van a correlacionar directamente con la selección de programas que van a ser usados, configurándose los móviles como espacios tan íntimos y personales que sus características van a depender, irremediablemente, de los intereses, las intenciones y las personalidades de cada persona usuaria (García y Monferrer, 2009). Así, es casi imposible hablar de lo positivo y lo negativo en el uso cotidiano de los smartphones sin contextualizar y personalizar cada experiencia de usuario. El móvil no forma parte de la actividad si no de la identidad de la persona, es parte de la construcción de un mundo personal y social que se evidencia en la relación vital con esta tecnología, que acaba por asumirse como estrategia (y no como aparato) para garantizar una presencia social y relacional en el mundo (Hoffner, Lee y Park, 2015) que responda a una serie de valores, creencias, significados y códigos.

\section{Método}

Este estudio se sitúa en el marco de una investigación con enfoque cualitativo con orientación a la innovación y transformación de la práctica docente (Fernández-Díaz y Calvo-Salvador, 2013) partiendo del concepto de investigación acción definido por Latorre (2003) como la indagación práctica realizada de manera colaborativa con el profesorado con el objetivo de mejorar su práctica educativa a través de dinámicas de acción y reflexión. La participación de profesionales de 12 centros públicos y concertados de entornos rurales y urbanos de Asturias fue necesaria, por tanto, para llevar a cabo esta propuesta de investigación. En total colaboramos con 30 profesores y profesoras de educación secundaria.

Los objetivos de este estudio en relación con las evidencias aquí planteadas son los siguientes:

- Conocer percepciones de los profesionales de la educación sobre Internet, redes sociales, telefonía móvil, etc., y su influencia en la vida cotidiana de las personas jóvenes.

- Profundizar en las opiniones y valoraciones tanto positivas como negativas que el colectivo de profesionales tiene sobre las TIC.

- Detectar necesidades demandadas por los y las profesionales de la educación en torno a las TIC como elementos mediadores de la socialización joven y de la construcción de identidades personales.

- Elaborar pautas y hacer propuestas para la reflexión, la formación y la intervención educativa en contextos formales reglados (centros educativos), orientadas hacia el uso crítico y activo de los medios de comunicación e información en el marco de las relaciones emocionales.

Para ello, se llevaron a cabo diferentes grupos focales dinamizados a partir de guiones abiertos de referencia, los cuales fueron modulados por el equipo de investigación siguiendo las normas de rigor. Tras la experiencia de cada grupo focal se realizó una detección de informantes clave con los que fuese posible tratar el tema en mayor profundidad para, posteriormente, aplicar con ese subgrupo entrevistas semiestructuradas. Estas entrevistas fueron guiadas con preguntas concretas basadas en 
el mismo guion que el trabajado anteriormente en los grupos focales. Cada entrevista semiestructurada fue personalizada, aplicando una propuesta de preguntas orientada a ahondar en torno a las respuestas ofrecidas previamente por cada persona en el grupo focal, de manera que en cada ocasión se incidió en aquellas cuestiones más fuertemente remarcadas con anterioridad.

La recogida de información se hizo a través de grabaciones en audio de las sesiones/entrevistas y posterior transcripción de las mismas. El proceso de análisis de los datos objetivos se centró en la descripción de categorías relevantes detectadas en función de los diferentes problemas específicos de estudio.

\section{Resultados}

Los resultados obtenidos en nuestro estudio se estructuran en torno a la gran categoría "Necesidades detectadas" y siguen el esquema de subcategorías presentado en la figura 1 y descritas a continuación.

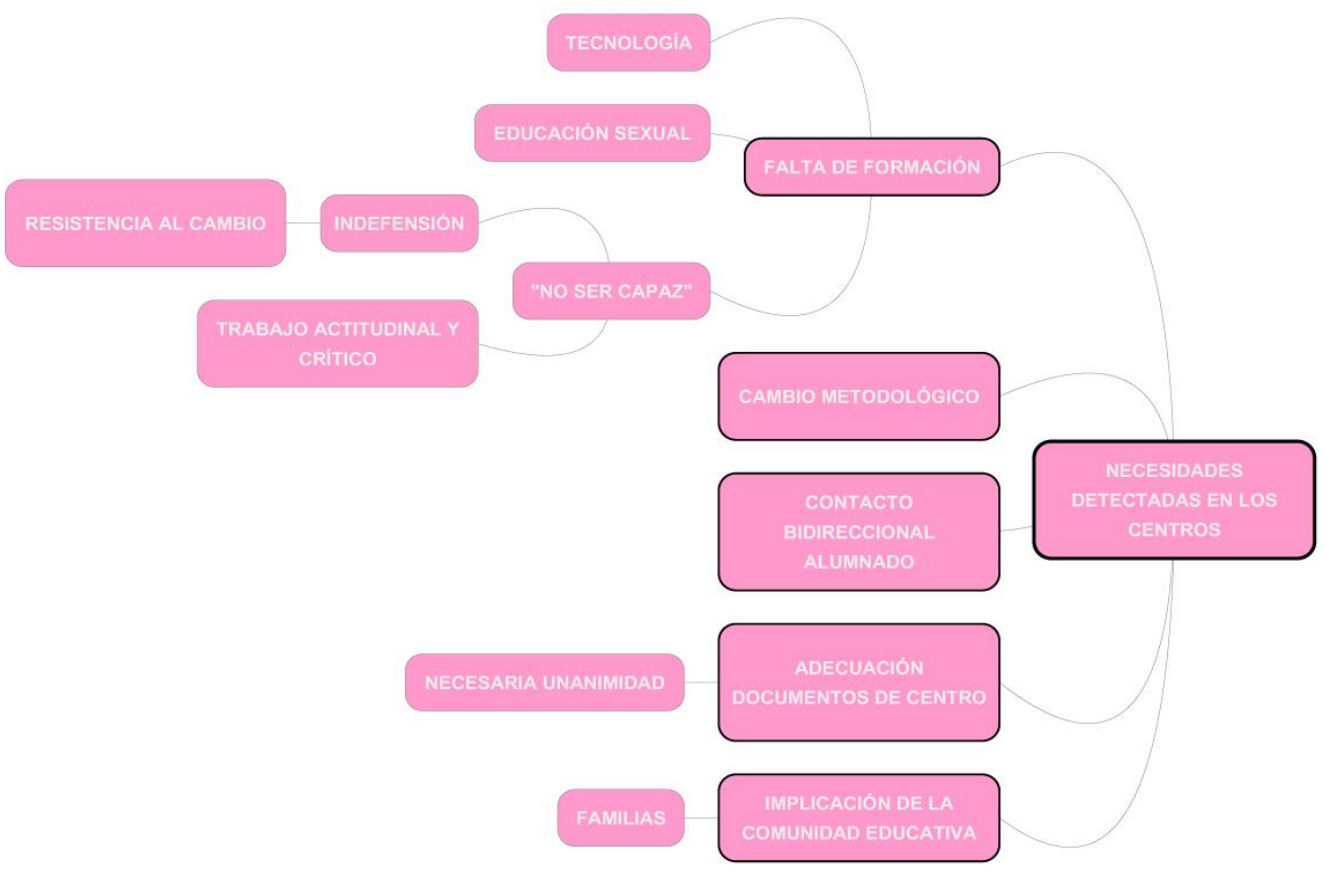

Figura 1. Mapa de categoría / subcategorías "Necesidades detectadas" Fuente: Elaboración propia.

\subsection{Subcategoría "Formación"}

La necesidad más evidente detectada por el profesorado participante es la falta de formación. En concreto, se demanda formación sobre tecnologías, educación sexual y relaciones emocionales. Uno de los puntos referentes de las necesidades formativas son las que tienen que ver con el abordamiento de las dificultades generadas en las vivencias cotidianas por los usos de las TIC. Es llamativa la necesidad expresada de trabajar cuestiones que tienen que ver con la práctica del sexting, en especial en lo que se refiere a información en torno a las cuestiones legales vinculadas. 
Yo creo que la información legal es lo primero, ¿no? (...) To creo que debería estar por escrito en todas las aulas. Hay cosas que tener presentes de continuo (...) Lo de los límites es importantísimo, para que ellos se puedan construir, para que puedan aprender, para que puedan desarrollarse emocionalmente y demás... los límites, el límite, un límite muy importante es el de la ley, evidentemente. (EN3)

Junto a la falta de formación es palpable la sensación de "no ser capaz" de enfrentarse a las problemáticas, siendo esta una tónica habitual que genera cierta indefensión y que acaba por ir de la mano de una resistencia al cambio. Se siente miedo e inseguridad; y esto acaba por paralizar iniciativas colectivas de intervención con los y las jóvenes. Se muestra poca inmersión profesional y personal en los espacios de socialización en línea; por lo que se desconocen sus particularidades.

Lo que pasa es que bueno, yo creo que tenemos un problema. A ver, estoy hablando desde mi situación. Creo que esto ha ido tan rápido, tan rápido, que muchos de nosotros necesitaríamos una alfabetización. Ellos han nacido ya con él, ¿̇ale? Han nacido ya en la era digital y han... vamos, lo han ido asimilando, están empapados, están imbuidos en ellos. (...) Es su lenguaje, es... y yo creo que es que, a ver, estoy insistiendo, desde mi perspectiva, desde mi edad, desde mi conocimiento van muy por delante de nosotros. (GF2, H6)

\subsection{Subcategorías "Cambio metodológico" / "Contacto con el alumnado"}

Los y las participantes requieren un trabajo actitudinal y crítico ya que muchos de los casos que suceden en los centros en relación con las tecnologías tienen más que ver con lo emocional que con lo académico. El cambio metodológico y el contacto bidireccional con el alumnado es considerado como imprescindible para poder enfrentarse a las necesidades de los centros y de las vivencias del alumnado de una manera constructiva y activa.

Punto de primera necesidad, porque forma parte de las formaciones de las identidades de futuros ciudadanos y ciudadanas, ciudadanas y ciudadanos. En el sentir, en el hacer, en el crearse una imagen de sí mismos y de sí mismas en el que elaborar un pensamiento crítico sobre lo que está ocurriendo tanto socialmente como conmigo misma. (...) los aspectos de las redes, porque son una segunda piel, es otro entorno que forma parte de mi piel. Entonces ahí es donde tenemos que trabajar, y no se está trabajando, y muchas competencias digitales, pero no se está trabajando en ello (...) tiene que ser un contenido a desarrollar, un contenido relevante (...) socio - educativo - emocional relevante. (EN1)

\subsection{Subcategoría "Documentos de centro"}

Esta falta de formación por parte de los equipos docentes de los centros tiene una repercusión en los documentos de centros y las normativas internas, de manera que no existen protocolos de actuación normalizados ante conflictos. Por ello, los y las profesionales no siempre saben cómo enfrentarse a las situaciones que se presentan en la convivencia dentro y fuera de las aulas. Por otra parte, no hay unanimidad, lo que es un hándicap social y educativo a tener en cuenta.

Creo que queda muy a título personal el resolver este tipo de problemas, se queda en la esfera del "qué haría yo", se toman decisiones por sentido común, no hay estrategias. (GF9)

\subsection{Subcategoría "Implicación de la comunidad educativa"}

Los y las profesionales demandan una mayor implicación por parte de las familias, de manera que pueda existir un trabajo colaborativo que envuelva a la comunidad educativa en general. Son significativas las alusiones que los y las participantes hacen acerca de las 
formas y los usos negativos de las TIC que son transmitidos desde padres y madres hasta hijos/as.

Cuando yo intervengo en problemas entre compañeros y de pareja, me encuentro con una situación que me choca mucho: Cómo percibe la familia los mensajes que se transmiten a través de las redes sociales. Hay una correspondencia entre cómo ven los jóvenes las cosas y en cómo las ven sus padres, los padres y sus hijos normalizan las mismas cosas. (GF5)

Se denuncia la intromisión de las familias en las gestiones internas y las normas que cada centro establece en torno a las prohibiciones o moderaciones sobre el uso de los móviles en el aula y en las instalaciones educativas.

$\Upsilon$ hay muchísimos padres, que de entrada te dicen: (...) "ustedes déjenme en paz que no tienen nada que meterse ustedes en ese tema. $\Upsilon$ yo a mi hija no le voy a quitar el Whatsapp. $\Upsilon$ yo a mi hija voy a seguir dejándole el teléfono. $\Upsilon$ ustedes dedíquense a dar clase que es lo que tienen que hacer." (...) hay como muy poco apoyo en ese sentido. (GF4, H1)

El profesorado considera que otro de los puntos cruciales que impide que estas situaciones puedan afrontarse de una manera positiva es la sensación de que las interacciones que suceden en las redes son asuntos "de fuera del centro", por lo que en muchas ocasiones es complejo convertirse en parte mediadora. A pesar de que los conflictos pueden sucederse fuera del horario escolar y de los límites geográficos de los centros tienen una repercusión clara en la convivencia escolar.

Lo que aquí dicen es que es fuera del centro, no, para nada, no es fuera del centro. La red tiene por lo que hemos dicho conexiones vitales, propicia una relación vital total día a día con lo que ocurre en el centro, día a día en la clase. (EN1)

\section{Discusión y conclusiones}

Gracias a los resultados obtenidos en nuestro estudio constatamos que existen dificultades generales por parte del profesorado para entender la realidad de la interacción digital de los jóvenes en términos emotivos y técnicos, tal y como apunta buena parte de la producción académica publicada en los últimos años (González, Gozálvez y Ramírez, 2015; Gozálvez y Aguaded, 2012). Estamos de acuerdo con Pérez y Rodríguez (2016) en que existe una tendencia por parte del profesorado de enseñanzas obligatorias de mostrar un déficit en el desarrollo de sus competencias didácticas para abordar los requerimientos socioculturales de la democratización cotidiana de las TIC. A pesar de estas barreras, detectamos unanimidad en torno a la idea de que las plataformas de socialización en línea son herramientas que forman parte de la realidad significativa del alumnado, y que por ello la escuela no puede obviarlas, debiendo responsabilizarse como parte activa de posibles soluciones.

El grupo de profesionales estudiado considera que el aprovechamiento didáctico de las TIC es cada vez más intenso. En contraposición con esta idea, se señala la desactualización de la legislación y la normativa interna de los centros; la cual no aborda la situación con rigurosidad. Esta idea refuerza las teorías que dibujan la relación compleja existente entre currículo educativo y TIC (George y Veytia, 2016), describiendo una disonancia entre la vida fuera y dentro de las paredes de la escuela, lo que puede derivar en rupturas educativo-contextuales. 
La necesidad de garantizar que la formación del profesorado supere el abordamiento de estas tecnologías desde lo meramente funcional y ahonde en la importancia que para los procesos emotivos e identitarios tiene la incorporación de estas herramientas de vinculación, se basa en un profundo concepto de equidad educativa tal y como lo plantean autoras como Anguita-Martínez (2011) o Sánchez-Santamaría y Ballester (2013). Sin una incorporación explícita del supuesto de equidad como base pedagógica indispensable no podríamos hablar de una educación en igualdad de oportunidades y posibilidades ya que la manera en que el profesorado se enfrenta a esta importante tarea obedece, en muchas ocasiones, al interés y la motivación personal. La situación de desigualdad que generan las diferentes maneras de entender lo afectivo como parte del concepto holístico de educación nos sitúa en un marco educativo injusto, que no puede por su propia naturaleza responder a las demandas globales del alumnado. Incorporar formalmente la necesidad de tratar estas cuestiones en el currículo educativo es imprescindible para materializar una educación centrada en ofrecer soluciones diversas para la vida en contextos plurales con intencionalidad inclusiva.

Nuestros datos arrojan evidencias en torno al papel de las tecnologías como mediadoras emocionales que sientan bases estratégicas y estructurales de relaciones significativas, enriqueciendo las líneas emergentes de debate existentes en torno a la aplicación de las TIC en los desarrollos socioafectivos (Colás-Bravo, González-Ramírez y de Pablos-Pons, 2013; Espinoza, 2015). Una educación holística no debería ignorar estas cuestiones si pretende atender a la globalidad del desarrollo personal. Las políticas escolares deberían replantearse para evitar la sensación de la existencia de espacios tecnológica y emocionalmente parcelados.

El profesorado denuncia la falta de apoyo por parte de la administración pública, lo que afecta a la formación continua y al desarrollo e implantación de programas específicos de intervención. Resaltan que no existe unanimidad en las políticas de los centros a la hora de enfocar el cambio actitudinal y metodológico requerido. También consideran como problemática la falta de trabajo en positivo, colaborativo con toda la comunidad educativa, y alejado de doctrinas alarmistas. Con estas conclusiones apoyamos las propuestas presentadas por Plaza y Caro (2016) en torno a la alfabetización digital de las familias como vía clave para favorecer una participación activa y cívica del alumnado; incidiendo positivamente en sus configuraciones identitaria y procesos de socialización.

Finalmente, nuestro estudio aboga por la creación de protocolos de actuación para los equipos de orientación y profesorado-tutor con el fin de establecer guías sólidas para la gestión de situaciones de conflicto derivadas del uso de las TIC, especialmente en relación con la mala gestión del sexting. Creemos imprescindible que la administración pública diseñe, en colaboración con personas expertas en género y educación sexual, líneas de intervención y propuestas de formación que permitan al profesorado ofrecer respuestas de utilidad ante este tipo de dificultades.

Entre las limitaciones de este estudio, destacamos el tipo de muestra participante; así como el plano contextual que, por su propia naturaleza, impide la generalización de los resultados. Como futuras líneas de investigación, creemos conveniente seguir trabajando sobre el sexting como nuevo ámbito de estudio en el campo de la intervención socioeducativa; así como en la necesidad de vincular currículo y política educativa a los cambios provocados por la sociedad red y su influencia en la construcción de identidades generizadas. 


\section{Referencias}

AIMC. (2017). Informe navegantes en la red 2017. Madrid: AIMC.

Almansa, A., Fonseca, O. y Castillo, A. (2013). Redes sociales y jóvenes. Uso de facebook en la juventud colombiana y española. Comunicar. Revista Científica de Comunicación y Educación, 2O(40), 127-135. https://doi.org/10.3916/C40-2013-03-03

Anguita-Martínez, R. (2011). El reto de la formación del profesorado para la igualdad. Revista Interuniversitaria de Formación del Profesorado, 14(1), 43-51.

Arriaga, A. y Marcellán Baraze, I. (2016). Las redes sociales: Espacios de participación y aprendizaje para la producción de imágenes digitales de los jóvenes. Estudios Sobre Educación, 30, 197-216. https://doi.org/10.15581/004.30.197-216

Aymerich-Franch, L. y Fedele, M. (2015). La implementación de los social media como recurso docente en la universidad presencial: La perspectiva de los estudiantes de comunicación. REICE. Revista Iberoamericana sobre Calidad, Eficacia y Cambio en Educación, 13(1), 19-33.

Ballesteros, J. C. y Megías, I. (2015). Jóvenes en la red: Un selfie. Madrid: Centro Reina Sofía sobre Adolescencia y Juventud.

Barrios, A. (2009). Los jóvenes y la red: usos y consumos de los nuevos medios en la sociedad de la información y la comunicación. Signo y Pensamiento, 28(54), 265-275.

Barujel, A. G., Varela, F. F. y Rodes, V. (2017). Niños y adolescentes frente a la competencia digital. Entre el teléfono móvil, youtubers y videojuegos. Revista Interuniversitaria de Formación del Profesorado, 89(31.2), 171-186.

Bernete, F. (2009). Usos de las TIC, Relaciones sociales y cambios en la socialización de las y los jóvenes. Revista de Estudios de Juventud, 88, 97-114.

Buckingham, D. y Martínez-Rodríguez, J. B. (2013). Jóvenes interactivos: Nueva ciudadanía entre redes sociales y escenarios escolares. Comunicar. Revista Científica de Comunicación y Educación, 20(40), 10-13. https://doi.org/10.3916/C40-2013-02-00

Caldevilla, D. (2010). Las redes sociales. Tipología, uso y consumo de las redes 2.0 en la sociedad digital actual. Documentación de las Ciencias de la Información, 33(1), 45-68.

Campos-Freire, F. (2008). Las redes sociales trastocan los modelos de los medios de comunicación tradicionales. Revista Latina de Comunicación Social, 63, 287-293.

Caro, L. (2015). WhatsApp y la búsqueda de control de la situación informacional por parte de los jóvenes. Sphera Publica, 15, 76-96.

Castells, M. (2006). La sociedad red. Madrid: Alianza Editorial.

Chóliz, M. (2012). Mobile-phone addiction in adolescence: The test of mobile phone dependence (TMD). Progress in Health Sciences, 2(1), 33-44.

Church, K. y de Oliveira, R. (2013). What's up with whatsapp? Comparing mobile instant messaging behaviors with traditional SMS. En MobileHCI '13 (Ed.), Proceedings of the 15th international conference on human-computer interaction with mobile devices and services (pp. 352361). Múnich: ACM Digital Library. https://doi.org/10.1145/2493190.2493225

Colás-Bravo, P., González-Ramírez, T. y de Pablos-Pons, J. (2013). Juventud y redes sociales: Motivaciones y usos preferentes. Comunicar. Revista Científica de Comunicación y Educación, 2O(40), 15-23. https://doi.org/10.3916/C40-2013-02-01 
Cuadrado, I., Martín-Mora, G., Antelo, F. I. y Merchán, I. M. (2014). Análisis metaperceptivo de los elementos expresivos de internet: clasificación y uso de emoticonos. Internet Latent Corpus Journal, 4(1), 29-45.

Cuesta, U. y Gaspar, S. (2013). Análisis motivacional del uso del smartphone entre jóvenes: Una investigación cualitativa. Historia y Comunicación Social, 18, 435-447.

Domínguez-Fernández, G., Jaén-Martínez, A. y Ceballos-García, M. J. (2017). Educar la virtualidad. Pixel-Bit. Revista de Medios $\Upsilon$ Educación, 50, 187-199. https://doi.org/10.12795/pixelbit.2017.i50.13

Espinoza, J. (2015). Reconfigurando el amor: Mediación tecnológica y relaciones afectivas. Question, I(45), 86-96. https://doi.org/10.2415/16696581

Fernández-Díaz, E. y Calvo-Salvador, A. (2013). Estrategias para la mejora de la práctica docente. Una investigación-acción colaborativa para el uso innovador de las TIC. Revista Electrónica Interuniversitaria de Formación del Profesorado, 16(2), 121-133.

García-Jiménez, A., López de Ayala-López, M. C. y Catalina-García, B. (2013). Hábitos de uso en Internet y en las redes sociales de los adolescentes españoles. Comunicar. Revista Científica de Comunicación y Educación, 41, 195-204. https://doi.org/10.3916/C41-2013-19

García-Martín, J. y García-Sánchez, J. N. (2015). Use of facebook, tuenti, twitter and myspace among young Spanish people. Behaviour y Information Technology, 34(7), 685-703. //doi.org/10.1080/0144929X.2014.993428

García, M. C. y Monferrer, J. (2009). Propuesta de análisis teórico sobre el uso del teléfono móvil en adolescentes. Comunicar. Revista Científica de Comunicación y Educación, 16(33), 83-92. https://doi.org/10.3916/c33-2009-02-008

Garmendia, M., Jiménez, E., Casado, M. A. y Mascheroni, G. (2016). Net children go mobile: Riesgos y oportunidades en internet y el uso de dispositivos móviles entre menores españoles (2010-2015). Madrid: Red.es y Universidad del País Vasco.

George, C. E. y Veytia, M. G. (2016). La difícil relación de las TIC y la innovación educativa con el currículum. Increscendo Educación y Humanidades, 3(2), 46-58.

Gómez del Castillo, M. T. (2017). Utilización de WhatsApp para la comunicación en titulados superiores. REICE. Revista Iberoamericana sobre Calidad, Eficacia y Cambio en Educación, 15(4), 51-65. https://doi.org/10.15366/reice2017.15.4.003

González, N., Gozálvez, V. y Ramírez, A. (2015). La competencia mediática en el profesorado no universitario. Diagnóstico y propuestas formativas. Revista de Educación, 367, 117-146. https://doi.org/ 10.4438/1988-592X-RE-2015-367-285.

Gozálvez, V. y Aguaded, J. I. (2012). Educación para la autonomía en sociedades mediáticas. Anàlisi: Quaderns de Comunicació I Cultura, 45, 1-14. https://doi.org/ 10.7238/a.voi45.1326

Graner, P., Beranuy, M., Sánchez-Carbonell, X., Castellanal, M. y Chamarro, A. (2007). ¿Qué uso hacen los jóvenes y adolescentes de internet y del móvil? En L. Álvarez Pousa (Ed.). Comunicación e xuventude: Actas do foro internacional (pp. 71-90). A Coruña: Colexio Profesional de Xornalistas de Galicia.

Hoffner, C. A., Lee, S. y Park, S. J. (2015). I miss my mobile phone! Self-expansion via mobile phone and responses to phone loss. New Media y Society, 18(11), 2452-2468. https://doi.org/10.1177/1461444815592665.

Hu, Y., Manikonda, L. y Kambhampati, S. (2014). What we instagram: A first analysis of instagram photo content and user types. En E. Adar (Comp.), Proceedings of the Eight International AAAI Conference on Weblogs and Social Media (pp. 595-598). Oxford: AAAI. 
IAB. (2017). VII estudio de redes sociales. Madrid: IAB.

Latorre, A. (2003). La investigación-acción. Conocer y cambiar la práctica educativa. Barcelona: Graó.

Lee, E., Lee, J. A., Moon, J. H. y Sung, Y. (2015). Pictures speak louder than Words: Motivations for using instagram. Cyberpsychology, Behavior and Social Networking, 18(9), 552-556. https://doi.org/10.1089/cyber.2015.0157

Livingstone, S., Haddon, L., Görzig, A. y Ólafsson, K. (2011). Risks and safety on the internet: The perspective of European children. Londres: EU Kids Online.

Madden, M., Lenhart, A. y Cortesi, S. (2013). Teens, social media, and privacy. Washington, DC: Pew Research Center.

Marcelino, G. V. (2015). Migración de los jóvenes españoles en redes sociales, de tuenti a facebook y de facebook a instagram. La segunda migración. ICONO14. Revista Científica de Comunicación y Tecnologías Emergentes, 13, 48-72. https://doi.org/10.7195/ri14.v13i2.821.

Merino, L. (2011). Jóvenes en redes sociales: Significados y prácticas de una sociabilidad digital. Revista de Estudios de Juventud, 95(1), 31-43.

Monsoriu, M. (2009). Manual de redes sociales en Internet. Aprende a usar tuenti, facebook, fotolog, myspace, etc. ;Mejor que tus hijos! Madrid: Creaciones Copyright.

ONTSI. (2017). Perfil sociodemográfico de los internautas. Análisis de datos INE 2016. Madrid: Ministerio de Industria, Energía y Turismo.

Plaza, J. y Caro, C. (2016). La implicación de la familia en la formación ético-cívica de los jóvenes a través de las TIC. Aloma: Revista de Psicologia, Ciències de L'educació I de L'esport Blanquerna, 34(2), 97-106.

Qiu, L., Lu, J., Yang, S., Qu, W. y Zhu, T. (2015). What does your selfie say about you? Computers in Human Behavior, 52, 443-449. https://doi.org/10.1016/j.chb.2015.06.032

Reguillo, R. (2012). Navegaciones errantes. De músicas, jóvenes y redes: De facebook a youtube y viceversa. Comunicación y Sociedad, 18, 135-171.

Reig, D. y Vílchez, L. (2013). Los jóvenes en la era de la hiperconectividad: Tendencias, claves y miradas. Madrid: Fundación Telefónica.

Rial, A., Gómez, P., Varela, J. y Braña, T. (2014). Actitudes, percepciones y uso de internet y las redes sociales entre los adolescentes de la comunidad gallega. Anales de Psicología, 30(2), 642-655. https://doi.org/10.6018/analesps.30.2.159111

Rubio-Romero, J. y Perlado, M. (2015). El fenómeno whatsapp en el contexto de la comunicación personal: Una aproximación a través de los jóvenes universitarios. ICONO14. Revista Científica de Comunicación y Tecnologías Emergentes, 13(2), 73-98. https://doi.org/10.7195/ri14.v13i2.818

Salomon, D. (2013). Moving on from facebook: Using instagram to connect with undergraduates and engage in teaching and learning. College y Research Libraries News, 74.(8), 408-412.

Sánchez-Burón, A. y Álvaro-Martín, A. (2010). Informe generación 2.0: Hábitos de los adolescentes en el uso de las redes sociales. Estudio comparativo entre comunidades autónomas. Madrid: Universidad Camilo José Cela.

Sánchez-Santamaría, J. y Ballester, M. G. (2013). Desarrollando el éxito educativo para todos: Reflexiones, propuestas y retos conceptuales en torno a la equidad educativa. REICE. Revista Iberoamericana Sobre Calidad, Eficacia y Cambio en Educación, 12(2), 85-104. 
Serrano-Puche, J. (2016). Internet y emociones: nuevas tendencias en un campo de investigación emergente. Comunicar. Revista Científica de Comunicación y Educación, 24(46), 19-26. https://doi.org/10.3916/C46-2016-02

Sixto, J. (2010). Comportamientos activos en usuarios 2.0: Facebook supera a tuenti, la red social que había sido líder en España. Causas, estrategias de comunicación e impacto en la recepción. Observatorio $\left(O B S^{*}\right), 4(2), 153-166$.

Sixto, J. (2011). La red social tuenti. Análisis del modelo de comunicación y de la estrategia de marketing. Cuadernos de Comunicación e Innovación, 89, 9-13.

Wood, A. F. y Smith, M. J. (2005). Online communication: Linking technology, identity y culture. Mahwah, NJ: Erlbaum.

\section{Breve CV de los autores}

\section{Soraya Calvo González}

Doctora en Equidad e Innovación en Educación (UniOvi, Unican, UVigo, USC y UDC) y acreditada como Profesora Ayudante Doctora por ANECA. Máster en Comunicación y Educación en la Red (UNED), Máster en Intervención e Investigación Socioeducativa (UniOvi) y Título Propio en Sexología (UAH). En la actualidad trabaja como Profesora Asociada en el departamento de Ciencias de la Educación de la Universidad de Oviedo y coordina el Servicio de Orientación e Información Sexual del Ayuntamiento de Gijón. Es profesora colaboradora en el Máster Oficial en Sexología de la UCJC y miembro del Grupo de Investigación "Análisis, Intervención y Evaluación Socioeducativa" (AIES) (http://aies.grupos.uniovi.es/). Su producción científica y divulgativa se centra en la educación sexual con perspectiva de género, la alfabetización mediática crítica y la innovación educativa. ORCID ID: 0000-0002-9596-3007. Email: calvosoraya@uniovi.es

\section{José Luis San Fabián}

Catedrático de Universidad en Didáctica y Organización Escolar, Titulado en Pedagogía y Sociología, investiga en los campos de Organización y Evaluación de Programas e Instituciones socioeducativas, Estudios sobre la Infancia, Investigación Cualitativa en Educación, publicando numerosos trabajos en revistas científicas. Ha dirigido 21 tesis doctorales. Premio Extraordinario de Doctorado por la Universidad Complutense de Madrid. Ha participado en más de 30 proyectos de investigación. Es Director del Programa de Doctorado en "Equidad e Innovación Educativa" en la Universidad de Oviedo. Es Director del Grupo de Investigación "Análisis, Intervención y Evaluación Socioeducativa” (AIES) (http://aies.grupos.uniovi.es/). Ha dirigido el Departamento de Ciencias de la Educación. Ha sido director de la Revista Organización y Gestión Educativa, del Forum Europeo de Administradores de la Educación. ORCID ID: 00000002-8149-7797. Email: jlsanfa@uniovi.es 